\title{
Caratheodory's approximation for a type of Caputo fractional stochastic differential equations
}

\author{
Zhongkai Guo', Junhao Hu* and Weifeng Wang ${ }^{1}$
}

\section{"Correspondence:}

junhaohu74@163.com

'School of Mathematics and Statistics, South-Central University for Nationalities, 430074, Wuhan, China

\begin{abstract}
The Caratheodory approximation for a type of Caputo fractional stochastic differential equations is considered. As is well known, under the Lipschitz and linear growth conditions, the existence and uniqueness of solutions for some type of differential equations can be established. However, this approach does not give an explicit expression for solutions; it is not applicable in practice sometimes. Therefore, it is important to seek the approximate solution. As an extending work for stochastic differential equations, in this paper, we consider Caratheodory's approximate solution for a type of Caputo fractional stochastic differential equations.
\end{abstract}

MSC: Primary 26A33; secondary $60 \mathrm{H} 10$

Keywords: Caputo derivative; Stochastic differential equation; Caratheodry's approximation

\section{Introduction}

Recently, stochastic fractional differential equations and stochastic fractional partial differential equations have attracted more and more attention. It turns out that differential equations involving derivatives of non-integer orders have memory properties, which are called non-local properties. Because of the non-local property of the Caputo fractional derivatives in time, Caputo fractional differential equations are important to model and describe problems in many disciplines, such as engineering, physics, and chemistry. For more details, see [1-7].

Compared with the work on deterministic fractional differential equations, the study of stochastic fractional differential equations is still in its infancy. However, the majority of work is concerned about the existence and uniqueness of solutions; see [8-12]. Until quite recently, there were some authors who considered some types of Caputo fractional stochastic differential equations and Caputo fractional stochastic partial differential equations by different approaching. For example, in Ref. [13], the authors considered the existence of stable manifolds for a type of stochastic differential equations. The authors of paper [14] considered the averaging principle of a type of stochastic fractional differential under some conditions consistent with the stochastic differential equations. In [15], the

(c) The Author(s) 2020. This article is licensed under a Creative Commons Attribution 4.0 International License, which permits use sharing, adaptation, distribution and reproduction in any medium or format, as long as you give appropriate credit to the original author(s) and the source, provide a link to the Creative Commons licence, and indicate if changes were made. The images or other third party material in this article are included in the article's Creative Commons licence, unless indicated otherwise in a credit line to the material. If material is not included in the article's Creative Commons licence and your intended use is not permitted by statutory regulation or exceeds the permitted use, you will need to obtain permission directly from the copyright holder. To view a copy of this licence, visit http://creativecommons.org/licenses/by/4.0/. 
existence of global forward attracting set for stochastic lattice systems with a Caputo fractional time derivative in the weak mean-square topology is established. In [16], the asymptotic distance between two distinct solutions is considered under a temporally weighted norm. Its worth mentioning that the Euler-Maruyama type approximate results for Caputo fractional stochastic differential equations have been established by [17]. For more related work, see [12, 18-22].

The Caratheodory approximation scheme was first considered by Caraheodory for ordinary differential equations, then Bell, Mohammad and Mao extended it to the stochastic differential equations case; see [23]. To the best of our knowledge, there is no work paying attention to the Caratheodory approximation for the Caputo fractional stochastic differential equation. In this paper, we will consider the Caratheodory approximation for the following type of Caputo fractional stochastic differential equation:

$$
\left\{\begin{array}{l}
D_{t}^{\alpha} X_{t}=f\left(t, X_{t}\right) d t+g\left(t, X_{t}\right) d B_{t}, \quad t \geq 0 \\
X_{0}=x_{0} \in L^{2}(\Omega, H)
\end{array}\right.
$$

where $\alpha \in\left(\frac{1}{2}, 1\right)$. For more details see Sect. 2. The aim of this paper is to extend the Caratheodory approximate results for Eq. (1.1).

This article is organized as follows. In Sect. 2 we will give some assumptions and basic results that we need. The existence and uniqueness of solution will be discussed in Sect. 3 . In the last section, we will consider the Caratheodory approximation for the Caputo fractional stochastic differential equations.

Throughout this paper, the letter $C$ will denote positive constants whose value may change in different occasions. We will write the dependence of a constant on parameters explicitly if it is essential.

\section{Preliminaries}

We impose the following assumptions to guarantee the existence and uniqueness of solution, $H$ denote a Hilbert space, its norm is denoted by $|\cdot|$.

H1: Lipschitz condition: Let $t \geq 0$ and constant $k>0$, such that, for all $x, y \in H$,

$$
|f(t, x)-f(t, y)|^{2}+|g(t, x)-g(t, y)|^{2} \leq k|x-y|^{2} .
$$

H2: Growth condition: Let $t \geq 0$ and constant $k>0$, such that, for all $x \in H$,

$$
|f(t, x)|^{2}+|g(t, x)|^{2} \leq k\left(1+|x|^{2}\right) .
$$

The following generalization of Gronwall's lemma for singular kernels is needed for us to establish our results; see [15, 24].

Lemma 2.1 Suppose $b \geq 0, \beta>0$ and $a(t)$ is a nonnegative function locally integrable on $0 \leq t<T$ (some $T \leq+\infty$ ), and suppose $u(t)$ is nonnegative and locally integrable on $0 \leq t<T$ with

$$
u(t) \leq a(t)+b \int_{0}^{t}(t-s)^{\beta-1} u(s) d s
$$


Then

$$
u(t) \leq a(t)+\int_{0}^{t}\left[\sum_{n=1}^{\infty} \frac{(b \Gamma(\beta))^{n}}{\Gamma(n \beta)}(t-s)^{n \beta-1} a(s)\right] d s, \quad 0 \leq t<T,
$$

where $\Gamma(\cdot)$ is the Gamma function.

\section{Well-posedness}

In this section, we consider the existence and uniqueness of solution for the following equation under conditions $\mathbf{H 1}$ and $\mathbf{H 2}$ :

$$
\left\{\begin{array}{l}
D_{t}^{\alpha} X_{t}=f\left(t, X_{t}\right) d t+g\left(t, X_{t}\right) d B_{t}, \quad t \geq 0, \frac{1}{2}<\alpha<1, \\
X_{0}=x_{0} \in L^{2}(\Omega, H)
\end{array}\right.
$$

where $B_{t}$ is a scalar Brownian motion, $f$ and $g$ are $H$-value functions.

Definition 3.1 An $H$-value $\mathcal{F}_{t}$-adapted stochastic process $X_{t}, t \in[0, T]$, is called a solution of the initial value problem (3.1), if $X_{t} \in C\left([0, T] ; L^{2}(\Omega, H)\right)$ and satisfies the following integral equation:

$$
\begin{aligned}
X_{t}= & x_{0}+\frac{1}{\Gamma(\alpha)} \int_{0}^{t}(t-s)^{\alpha-1} f\left(s, X_{s}\right) d s \\
& +\frac{1}{\Gamma(\alpha)} \int_{0}^{t}(t-s)^{\alpha-1} g\left(s, X_{s}\right) d B_{s} .
\end{aligned}
$$

The existence and uniqueness of solutions for Eq. (3.1) have been considered by our previous work [25]. Similar problem also considered by [16] under different framework. To make this paper self-contained, we just give the main part of the proof for the following theorem.

Theorem 3.1 ([25]) Under conditions $\mathbf{H 1}$ and $\mathbf{H 2}$, for every $x_{0} \in L^{2}(\Omega, H)$, Eq. (3.1) has a unique mild solution $X_{t} \in C\left([0, T] ; L^{2}(\Omega, H)\right)$.

Proof We prove the theorem by the contraction mapping principle. Using conditions H1 and H2, Lemma 2.1, we can derive that $X_{t} \in C\left([0, T] ; L^{2}(\Omega, H)\right)$.

Let

$$
S=\left\{X_{t} \mid X_{t} \in C\left([0, T] ; L^{2}(\Omega, H)\right)\right\}
$$

equipped with the norm

$$
|f(t)|_{\varsigma}=\sup _{0 \leq t \leq T} E|f(t)|^{2}
$$

be the Banach space of all $\mathcal{F}_{t}$-adapted processes.

For any $t \in[0, T]$ and $X_{t} \in S$, define a mapping as follows:

$$
(\Phi X)(t)=x_{0}+\frac{1}{\Gamma(\alpha)} \int_{0}^{t}(t-s)^{\alpha-1} f\left(s, X_{s}\right) d s+\frac{1}{\Gamma(\alpha)} \int_{0}^{t}(t-s)^{\alpha-1} g\left(s, X_{s}\right) d B_{s} .
$$


It is easy to verify that

$$
\Phi(\cdot): C\left([0, T], L^{2}(\Omega ; H)\right) \rightarrow C\left([0, T], L^{2}(\Omega ; H)\right)
$$

Let $X_{t}, Y_{t} \in S$, then

$$
\begin{aligned}
E|(\Phi X)(t)-(\Phi Y)(t)|^{2} \leq & 2 E\left|\frac{1}{\Gamma(\alpha)} \int_{0}^{t}(t-s)^{\alpha-1}\left[f\left(s, X_{s}\right)-f\left(s, Y_{s}\right)\right] d s\right|^{2} \\
& +2 E\left|\frac{1}{\Gamma(\alpha)} \int_{0}^{t}(t-s)^{\alpha-1}\left[g\left(s, X_{s}\right)-g\left(s, Y_{s}\right)\right] d B_{s}\right|^{2} .
\end{aligned}
$$

Denote $\beta=2 \alpha-1>0$, by the Cauchy-Schwartz inequality, Itô's isometry formula and condition $\mathbf{H 1}$, we have

$$
E|(\Phi X)(t)-(\Phi Y)(t)|^{2} \leq \frac{2 k(T+1)}{\Gamma(\alpha)^{2}} \int_{0}^{t}(t-s)^{\beta-1} E\left|X_{s}-Y_{s}\right|^{2} d s
$$

Using mathematical induction methods, we can deduce the following fact:

$$
E\left|\left(\Phi^{n} X\right)(t)-\left(\Phi^{n} Y\right)(t)\right|^{2} \leq \frac{1}{\beta}\left(\frac{2 k(T+1)}{\Gamma(\alpha)^{2}}\right)^{n} \frac{\Gamma(\beta)^{n}}{\Gamma(n \beta)} t^{n \beta}\left|X_{t}-Y_{t}\right|_{\varsigma} .
$$

For $n=1$, by simple calculation we get

$$
E|(\Phi X)(t)-(\Phi Y)(t)|^{2} \leq \frac{2 k(T+1)}{\Gamma(\alpha)^{2}}\left|X_{t}-Y_{t}\right|_{\varsigma} \frac{t^{\beta}}{\beta}
$$

which satisfies Eq. (3.3) with $n=1$.

Now, assuming that Eq. (3.3) is satisfied for $n=j$, we claim that it is also correct for $n=j+1$. We have

$$
\begin{aligned}
E \mid & \left(\Phi^{j+1} X\right)(t)-\left.\left(\Phi^{j+1} Y\right)(t)\right|^{2} \\
& \leq \frac{2 k(T+1)}{\Gamma(\alpha)^{2}} \int_{0}^{t}(t-s)^{\beta-1} E\left|\left(\Phi^{j} X\right)(s)-\left(\Phi^{j} Y\right)(s)\right|^{2} d s \\
& \leq \frac{2 k(T+1)}{\Gamma(\alpha)^{2}} \int_{0}^{t}(t-s)^{\beta-1} \frac{1}{\beta}\left(\frac{2 k(T+1)}{\Gamma(\alpha)^{2}}\right)^{j} \frac{\Gamma(\beta)^{j}}{\Gamma(j \beta)} s^{j \beta}\left|X_{s}-Y_{s}\right|_{\varsigma} d s \\
& \leq\left(\frac{2 k(T+1)}{\Gamma(\alpha)^{2}}\right)^{j+1} \frac{1}{\beta} \frac{\Gamma(\beta)^{j}}{\Gamma(j \beta)}\left|X_{t}-Y_{t}\right|_{\varsigma} \int_{0}^{t}(t-s)^{\beta-1} s^{j \beta} d s .
\end{aligned}
$$

To get the estimate for $n=j+1$, we only need to consider the following integral:

$$
\int_{0}^{t}(t-s)^{\beta-1} s^{j \beta} d s
$$

Take $s=t z$, then

$$
\begin{aligned}
\int_{0}^{t}(t-s)^{\beta-1} s^{j \beta} d s & =\int_{0}^{1}(1-z)^{\beta-1} t^{\beta-1} t^{j \beta} z^{j \beta} t d z \\
& =t^{(j+1) \beta} \int_{0}^{1}(1-z)^{\beta-1} z^{j \beta} d z
\end{aligned}
$$




$$
\begin{aligned}
& =t^{(j+1) \beta} B(j \beta+1, \beta) \\
& =t^{(j+1) \beta} \frac{\Gamma(\beta) \Gamma(j \beta+1)}{\Gamma((j+1) \beta+1)},
\end{aligned}
$$

where $B(\cdot, \cdot)$ is the Beta function. Combining this result with Eq. (3.4) we have

$$
\begin{aligned}
E \mid & \left(\Phi^{j+1} X\right)(t)-\left.\left(\Phi^{j+1} Y\right)(t)\right|^{2} \\
& \leq\left(\frac{2 k(T+1)}{\Gamma(\alpha)^{2}}\right)^{j+1} \frac{1}{\beta} \frac{\Gamma(\beta)^{j}}{\Gamma(j \beta)}\left|X_{t}-Y_{t}\right|_{\varsigma} t^{(j+1) \beta} \frac{\Gamma(\beta) \Gamma(j \beta+1)}{\Gamma((j+1) \beta+1)} \\
& =\left(\frac{2 k(T+1)}{\Gamma(\alpha)^{2}}\right)^{j+1} \frac{1}{\beta} \Gamma(\beta)^{j+1} \frac{\Gamma(j \beta+1)}{\Gamma((j+1) \beta+1) \Gamma(j \beta)} t^{(j+1) \beta}\left|X_{t}-Y_{t}\right|_{\varsigma} \\
& =\left(\frac{2 k(T+1)}{\Gamma(\alpha)^{2}}\right)^{j+1} \frac{1}{\beta} \Gamma(\beta)^{j+1} \frac{j \beta \Gamma(j \beta)}{(j+1) \beta \Gamma(j+1) \beta) \Gamma(j \beta)} t^{(j+1) \beta}\left|X_{t}-Y_{t}\right|_{\varsigma} \\
& \leq\left(\frac{2 k(T+1)}{\Gamma(\alpha)^{2}}\right)^{j+1} \frac{1}{\beta} \Gamma(\beta)^{j+1} \frac{t^{(k+1) \beta}}{\Gamma((j+1) \beta)}\left|X_{t}-Y_{t}\right|_{\varsigma} .
\end{aligned}
$$

Then we arrive at the following estimate for all $n$ :

$$
\left|\left(\Phi^{n} X\right)(t)-\left(\Phi^{n} Y\right)(t)\right|_{\varsigma} \leq\left(\frac{2 k(T+1)}{\Gamma(\alpha)^{2}}\right)^{n} \frac{1}{\beta} \Gamma(\beta)^{n} \frac{T^{n \beta}}{\Gamma(n \beta)}\left|X_{t}-Y_{t}\right|_{\varsigma} .
$$

If we can prove

$$
\left(\frac{2 k(T+1)}{\Gamma(\alpha)^{2}}\right)^{n} \frac{1}{\beta} \Gamma(\beta)^{n} \frac{T^{n \beta}}{\Gamma(n \beta)}<1
$$

for sufficient large $n$, then the theorem holds.

Consider the following series of positive terms:

$$
\sum_{n=1}^{\infty}\left(\frac{2 k(T+1)}{\Gamma(\alpha)^{2}}\right)^{n} \frac{1}{\beta} \Gamma(\beta)^{n} \frac{T^{n \beta}}{\Gamma(n \beta)}
$$

We will show that

$$
\left(\frac{2 k(T+1)}{\Gamma(\alpha)^{2}}\right)^{n} \frac{1}{\beta} \Gamma(\beta)^{n} \frac{T^{n \beta}}{\Gamma(n \beta)} \rightarrow 0
$$

as $n \rightarrow+\infty$, which guarantees that Eq. (3.7) holds. Thanks to the d'Alembert discriminant method, we only need to justify

$$
\lim _{n \rightarrow \infty} \frac{\left(\frac{2 k(T+1)}{\Gamma(\alpha)^{2}}\right) \Gamma(\beta) T^{\beta} \Gamma(n \beta)}{\Gamma((n+1) \beta))}<1
$$

Use the relationship of Gamma function and the Stirling formula, represented as follows:

$$
\Gamma(x) \sim \sqrt{2 \pi} e^{-x} x^{x-\frac{1}{2}}, x \rightarrow \infty .
$$


Then

$$
\begin{aligned}
\lim _{n \rightarrow \infty} & \frac{\left(\frac{2 k(T+1)}{\Gamma(\alpha)^{2}}\right) \Gamma(\beta) T^{\beta} \Gamma(n \beta)}{\Gamma((n+1) \beta))} \\
= & \lim _{n \rightarrow \infty}\left(\frac{2 k(T+1)}{\Gamma(\alpha)^{2}}\right) \Gamma(\beta) T^{\beta} e^{\beta} \sqrt{\frac{n+1}{n}}\left(\frac{n}{n+1}\right)^{n \beta} \frac{1}{(n \beta+\beta)^{\beta}}=0,
\end{aligned}
$$

which shows that $\Phi(\cdot)$ is a contraction mapping on $C\left([0, T], L^{2}(\Omega ; H)\right)$ for all $T<\infty$. This completes the proof.

\section{Caratheodory's approximate solutions}

In this section, we consider the Caratheodory approximation for stochastic fractional differential equations. Similar to the stochastic differential equations approach, we try to give the definition of Caratheodory's approximate solutions for stochastic fractional differential equations as follows.

For every integer $n \geq 1$, define $x_{n}(t)=x_{0}$ for $-1 \leq t \leq 0$ and

$$
\begin{aligned}
x_{n}(t)= & x_{0}+\frac{1}{\Gamma(\alpha)} \int_{0}^{t}(t-s)^{\alpha-1} f\left(s, x_{n}\left(s-\frac{1}{n}\right)\right) d s \\
& +\frac{1}{\Gamma(\alpha)} \int_{0}^{t}(t-s)^{\alpha-1} g\left(s, x_{n}\left(s-\frac{1}{n}\right)\right) d B_{s}
\end{aligned}
$$

for $0<t \leq T$.

Note that, for $0 \leq t \leq \frac{1}{n}, x_{n}(t)$ can be computed by

$$
x_{n}(t)=x_{0}+\frac{1}{\Gamma(\alpha)} \int_{0}^{t}(t-s)^{\alpha-1} f\left(s, x_{0}\right) d s+\frac{1}{\Gamma(\alpha)} \int_{0}^{t}(t-s)^{\alpha-1} g\left(s, x_{0}\right) d B_{s},
$$

then, for $\frac{1}{n}<t \leq \frac{2}{n}$,

$$
\begin{aligned}
x_{n}(t)= & x_{n}\left(\frac{1}{n}\right)+\frac{1}{\Gamma(\alpha)} \int_{\frac{1}{n}}^{t}(t-s)^{\alpha-1} f\left(s, x_{n}\left(s-\frac{1}{n}\right)\right) d s \\
& +\frac{1}{\Gamma(\alpha)} \int_{\frac{1}{n}}^{t}(t-s)^{\alpha-1} g\left(s, x_{n}\left(s-\frac{1}{n}\right)\right) d B_{s}
\end{aligned}
$$

and so on. By this approach, we can compute $x_{n}(t)$ step by step on the intervals $\left[0, \frac{1}{n}\right]$, $\left(\frac{1}{n}, \frac{2}{n}\right], \ldots$

Lemma 4.1 Under the condition $\mathbf{H 2}$, for all $n \leq 1$, we have

$$
\sup _{0 \leq t \leq T} E\left|x_{n}(t)\right|^{2} \leq \Omega=: r_{1}\left(1+E_{2 \alpha-1,1}\left(r_{2} \Gamma(2 \alpha-1) T^{2 \alpha-1}\right)\right)<\infty
$$

where $r_{1}=3 E\left|x_{0}\right|^{2}+3 \frac{\left(k T^{(2 \alpha-1)}\right)(T+1)}{\Gamma(\alpha)^{2}(2 \alpha-1)}, r_{2}=3 \frac{k(T+1)}{\Gamma(\alpha)^{2}}$ and $E_{2 \alpha-1,1}(\cdot)$ is a two-parameter function of the Mittag-Leffler type (see [15]).

Proof From the simple arithmetic inequality

$$
|a+b+c|^{2} \leq 3\left(|a|^{2}+|b|^{2}+|c|^{2}\right)
$$


we have

$$
\begin{aligned}
E\left|x_{n}(t)\right|^{2} \leq & 3 E\left|x_{0}\right|^{2}+3 E\left|\frac{1}{\Gamma(\alpha)} \int_{0}^{t}(t-s)^{\alpha-1} f\left(s, x_{n}\left(s-\frac{1}{n}\right)\right) d s\right|^{2} \\
& +3 E\left|\frac{1}{\Gamma(\alpha)} \int_{0}^{t}(t-s)^{\alpha-1} g\left(s, x_{n}\left(s-\frac{1}{n}\right)\right) d B_{s}\right|^{2} \\
:= & 3 I_{1}+3 I_{2}+3 I_{3} .
\end{aligned}
$$

By the Cauchy-Schwarz inequality and condition $\mathbf{H} 2$, we can estimate the term $I_{2}$ as follows:

$$
\begin{aligned}
I_{2} & \leq \frac{T k}{\Gamma(\alpha)^{2}} \int_{0}^{t}(t-s)^{2(\alpha-1)}\left(1+E\left|x_{n}\left(s-\frac{1}{n}\right)\right|^{2}\right) d s \\
& \leq \frac{T k}{\Gamma(\alpha)^{2}}\left[\frac{t^{2 \alpha-1}}{2 \alpha-1}+\int_{0}^{t}(t-s)^{2(\alpha-1)} E\left|x_{n}\left(s-\frac{1}{n}\right)\right|^{2} d s\right] \\
& \leq \frac{k T^{2 \alpha}}{\Gamma(\alpha)^{2}(2 \alpha-1)}+\frac{T k}{\Gamma(\alpha)^{2}} \int_{0}^{t}(t-s)^{2(\alpha-1)} \sup _{0 \leq r \leq s} E\left|x_{n}(r)\right|^{2} d s .
\end{aligned}
$$

Similarly, with Itô's isometry formula and condition H2, we have an estimate for the stochastic integral term:

$$
\begin{aligned}
I_{3} & \leq \frac{k}{\Gamma(\alpha)^{2}} \int_{0}^{t}(t-s)^{2(\alpha-1)}\left(1+E\left|x_{n}\left(s-\frac{1}{n}\right)\right|^{2}\right) d s \\
& \leq \frac{k T^{2 \alpha-1}}{\Gamma(\alpha)^{2}(2 \alpha-1)}+\frac{k}{\Gamma(\alpha)^{2}} \int_{0}^{t}(t-s)^{2(\alpha-1)} \sup _{0 \leq r \leq s} E\left|x_{n}(r)\right|^{2} d s .
\end{aligned}
$$

Combining the estimate for $I_{1}, I_{2}, I_{3}$, we arrive at

$$
E\left|x_{n}(t)\right|^{2} \leq r_{1}+r_{2} \int_{0}^{t}(t-s)^{(2 \alpha-1)-1} \sup _{0 \leq r \leq s} E\left|x_{n}(r)\right|^{2} d s,
$$

where we denote

$$
r_{1}=3 E\left|x_{0}\right|^{2}+3 \frac{\left(k T^{2 \alpha-1}\right)(T+1)}{\Gamma(\alpha)^{2}(2 \alpha-1)}
$$

and

$$
r_{2}=3 \frac{k(T+1)}{\Gamma(\alpha)^{2}} .
$$

Note that, for $t_{1} \leq t_{2}$, we have

$$
\int_{0}^{t_{1}}\left(t_{1}-s\right)^{(2 \alpha-1)-1} \sup _{0 \leq r \leq s} E\left|x_{n}(r)\right|^{2} d s \leq \int_{0}^{t_{2}}\left(t_{2}-s\right)^{(2 \alpha-1)-1} \sup _{0 \leq r \leq s} E\left|x_{n}(r)\right|^{2} d s .
$$

Then

$$
\sup _{0 \leq r \leq t} E\left|x_{n}(r)\right|^{2} \leq r_{1}+r_{2} \int_{0}^{t}(t-s)^{(2 \alpha-1)-1} \sup _{0 \leq r \leq s} E\left|x_{n}(r)\right|^{2} d s .
$$


Applying Lemma 2.1, we can directly obtain

$$
\begin{aligned}
\sup _{0 \leq r \leq t} E\left|x_{n}(r)\right|^{2} & \leq r_{1}\left(1+\int_{0}^{t} \sum_{n=1}^{\infty} \frac{\left(r_{2} \Gamma(2 \alpha-1)\right)^{n}}{\Gamma(2 n \alpha-n)}(t-s)^{n(2 \alpha-1)-1} d s\right) \\
& \leq r_{1}\left(1+\sum_{n=1}^{\infty} \frac{\left(r_{2} \Gamma(2 \alpha-1) T^{2 \alpha-1}\right)^{n}}{\Gamma(2 n \alpha-n+1)}\right) \\
& =r_{1}\left(1+E_{2 \alpha-1,1}\left(r_{2} \Gamma(2 \alpha-1) T^{2 \alpha-1}\right)\right)<\infty,
\end{aligned}
$$

for all $t \in[0, T]$, where $E_{2 \alpha-1,1}(\cdot)$ is a two-parameter function of the Mittag-Leffler type (see [15]).

Lemma 4.2 Under the condition $\mathbf{H 2}$, for all $n \geq 1$ and $0 \leq t_{0}<t \leq T$ with $t-t_{0} \leq 1$, then

$$
E\left|x_{n}(t)-x_{n}\left(t_{0}\right)\right|^{2} \leq C\left(t-t_{0}\right)^{2 \alpha-1}
$$

Proof Taking $0 \leq t_{0}<t \leq T$, we note that

$$
\begin{aligned}
E\left|x_{n}(t)-x_{n}\left(t_{0}\right)\right|^{2} & \\
\leq & 2 E \frac{1}{\Gamma(\alpha)^{2}}\left|\int_{0}^{t}(t-s)^{\alpha-1} f\left(s, x_{n}\left(s-\frac{1}{n}\right)\right) d s-\int_{0}^{t_{0}}\left(t_{0}-s\right)^{\alpha-1} f\left(s, x_{n}\left(s-\frac{1}{n}\right)\right) d s\right|^{2} \\
& +2 E \frac{1}{\Gamma(\alpha)^{2}} \mid \int_{0}^{t}(t-s)^{\alpha-1} g\left(s, x_{n}\left(s-\frac{1}{n}\right)\right) d B_{s} \\
& \quad-\left.\int_{0}^{t_{0}}\left(t_{0}-s\right)^{\alpha-1} g\left(s, x_{n}\left(s-\frac{1}{n}\right)\right) d B_{s}\right|^{2} \\
=: & 2\left(J_{1}+J_{2}\right) .
\end{aligned}
$$

For $J_{1}$, we have

$$
\begin{aligned}
J_{1} \leq & 2 E \frac{1}{\Gamma(\alpha)^{2}}\left|\int_{t_{0}}^{t}(t-s)^{\alpha-1} f\left(s, x_{n}\left(s-\frac{1}{n}\right)\right) d s\right|^{2} \\
& +2 E \frac{1}{\Gamma(\alpha)^{2}}\left|\int_{0}^{t_{0}}\left[(t-s)^{\alpha-1}-\left(t_{0}-s\right)^{\alpha-1}\right] f\left(s, x_{n}\left(s-\frac{1}{n}\right)\right) d s\right|^{2} \\
= & 2 J_{11}+2 J_{12} .
\end{aligned}
$$

Using the Cauchy-Schwartz inequality, $t-t_{0} \leq 1$, we give an estimate for $J_{11}$ as follows:

$$
\begin{aligned}
J_{11} & \leq \frac{1}{\Gamma(\alpha)^{2}} \int_{t_{0}}^{t}(t-s)^{2 \alpha-2} d s \int_{t_{0}}^{t} E\left|f\left(s, x_{n}\left(s-\frac{1}{n}\right)\right)\right|^{2} d s \\
& \leq \frac{k}{\Gamma(\alpha)^{2}(2 \alpha-1)}\left(t-t_{0}\right)^{2 \alpha-1} \int_{t_{0}}^{t}\left[1+E\left|x_{n}\left(s-\frac{1}{n}\right)\right|^{2}\right] d s \\
& \leq \frac{(\Omega+1) k}{\Gamma(\alpha)^{2}(2 \alpha-1)}\left(t-t_{0}\right)^{2 \alpha-1}
\end{aligned}
$$


where

$$
\Omega=r_{1}\left(1+E_{2 \alpha-1,1}\left(r_{2} \Gamma(2 \alpha-1) T^{2 \alpha-1}\right)\right)
$$

has been defined in Lemma 4.1.

For $J_{12}$, we have the following result:

$$
\begin{aligned}
J_{12} & =E \frac{1}{\Gamma(\alpha)^{2}}\left|\int_{0}^{t_{0}}\left[(t-s)^{\alpha-1}-\left(t_{0}-s\right)^{\alpha-1}\right] f\left(s, x_{n}\left(s-\frac{1}{n}\right)\right) d s\right|^{2} \\
& \leq \frac{k}{\Gamma(\alpha)^{2}} \int_{0}^{t_{0}}\left[(t-s)^{\alpha-1}-\left(t_{0}-s\right)^{\alpha-1}\right]^{2} d s \int_{0}^{t_{0}}\left[1+E\left|x_{n}\left(s-\frac{1}{n}\right)\right|^{2}\right] d s \\
& \leq \frac{C T k}{\Gamma(\alpha)^{2}} \int_{0}^{t_{0}}\left[\left(t_{0}-s\right)^{2 \alpha-2}-(t-s)^{2 \alpha-2}\right] d s \\
& =\frac{C T k}{\Gamma(\alpha)^{2}}\left[\frac{\left(t-t_{0}\right)^{2 \alpha-1}}{2 \alpha-1}+\frac{t_{0}^{2 \alpha-1}}{2 \alpha-1}-\frac{t^{2 \alpha-1}}{2 \alpha-1}\right] \\
& \leq \frac{C T k}{\Gamma(\alpha)^{2}} \frac{\left(t-t_{0}\right)^{2 \alpha-1}}{2 \alpha-1} .
\end{aligned}
$$

For $J_{2}$, taking the Itô isometry formula and condition $\mathbf{H 2}$ into account, using similar estimate methods to $J_{1}$, it can be shown that

$$
J_{2} \leq \frac{C k}{\Gamma(\alpha)^{2}} \frac{\left(t-t_{0}\right)^{2 \alpha-1}}{2 \alpha-1} .
$$

Combining all the deduced estimates, we have

$$
E\left|x_{n}(t)-x_{n}\left(t_{0}\right)\right|^{2} \leq C\left(t-t_{0}\right)^{2 \alpha-1} .
$$

This completes the proof.

Theorem 4.1 Under the conditions $\mathbf{H 1}$ and $\mathbf{H 2}$, let $x(t)$ be the unique solution of equations

(3.1). Then for $n \geq 1$

$$
\sup _{0 \leq t \leq T} E\left|x(t)-x_{n}(t)\right|^{2} \leq \frac{C}{n^{2 \alpha-1}}
$$

Proof Note that

$$
\begin{aligned}
x(t)-x_{n}(t)= & \frac{1}{\Gamma(\alpha)}\left[\int_{0}^{t}(t-s)^{\alpha-1} f(s, x(s)) d s-\int_{0}^{t}(t-s)^{\alpha-1} f\left(s, x_{n}\left(s-\frac{1}{n}\right)\right) d s\right] \\
& +\frac{1}{\Gamma(\alpha)}\left[\int_{0}^{t}(t-s)^{\alpha-1} g(s, x(s)) d B_{s}-\int_{0}^{t}(t-s)^{\alpha-1} g\left(s, x_{n}\left(s-\frac{1}{n}\right)\right) d B_{s}\right] .
\end{aligned}
$$

Hence, employing a simple arithmetic inequality, we have

$$
\begin{aligned}
& E\left|x(t)-x_{n}(t)\right|^{2} \\
& \quad \leq 2 E \frac{1}{\Gamma(\alpha)^{2}}\left|\int_{0}^{t}(t-s)^{\alpha-1} f(s, x(s)) d s-\int_{0}^{t}(t-s)^{\alpha-1} f\left(s, x_{n}\left(s-\frac{1}{n}\right)\right) d s\right|^{2}
\end{aligned}
$$




$$
\begin{aligned}
& +2 E \frac{1}{\Gamma(\alpha)^{2}}\left|\int_{0}^{t}(t-s)^{\alpha-1} g(s, x(s)) d B_{s}-\int_{0}^{t}(t-s)^{\alpha-1} g\left(s, x_{n}\left(s-\frac{1}{n}\right)\right) d B_{s}\right|^{2} \\
= & : 2\left(I_{1}+I_{2}\right) .
\end{aligned}
$$

For $I_{1}$, we have

$$
\begin{aligned}
I_{1} \leq & 2 E \frac{1}{\Gamma(\alpha)^{2}}\left|\int_{0}^{t}(t-s)^{\alpha-1} f(s, x(s)) d s-\int_{0}^{t}(t-s)^{\alpha-1} f\left(s, x_{n}(s)\right) d s\right|^{2} \\
& +2 E \frac{1}{\Gamma(\alpha)^{2}} \int_{0}^{t}(t-s)^{\alpha-1} f\left(s, x_{n}(s)\right) d s-\left.\int_{0}^{t}(t-s)^{\alpha-1} f\left(s, x_{n}\left(s-\frac{1}{n}\right)\right) d s\right|^{2} \\
= & : 2\left(I_{11}+I_{12}\right) .
\end{aligned}
$$

Using the Cauchy-Schwartz inequality and the condition H1, we have the following estimate for $I_{11}$ :

$$
I_{11} \leq \frac{k T}{\Gamma(\alpha)^{2}} \int_{0}^{t}(t-s)^{2 \alpha-2} E\left|x(s)-x_{n}(s)\right|^{2} d s
$$

Similarly, for $I_{12}$, we have

$$
I_{12} \leq \frac{k T^{2 \alpha-1}}{(2 \alpha-1) \Gamma(\alpha)^{2}} \int_{0}^{t} E\left|x_{n}(s)-x_{n}\left(s-\frac{1}{n}\right)\right|^{2} d s .
$$

Also, we can divide $I_{2}$ into two parts as follows:

$$
\begin{aligned}
I_{2} \leq & 2 E \frac{1}{\Gamma(\alpha)^{2}}\left|\int_{0}^{t}(t-s)^{\alpha-1} g(s, x(s)) d s-\int_{0}^{t}(t-s)^{\alpha-1} g\left(s, x_{n}(s)\right) d B_{s}\right|^{2} \\
& +2 E \frac{1}{\Gamma(\alpha)^{2}} \int_{0}^{t}(t-s)^{\alpha-1} g\left(s, x_{n}(s)\right) d s-\left.\int_{0}^{t}(t-s)^{\alpha-1} g\left(s, x_{n}\left(s-\frac{1}{n}\right)\right) d B_{s}\right|^{2} \\
= & 2\left(I_{21}+I_{21}\right) .
\end{aligned}
$$

By the Itô isometry formula, we get

$$
I_{21} \leq \frac{k}{\Gamma(\alpha)^{2}} \int_{0}^{t}(t-s)^{2 \alpha-2} E\left|x(s)-x_{n}(s)\right|^{2} d s
$$

and

$$
I_{22} \leq \frac{k}{\Gamma(\alpha)^{2}} \int_{0}^{t}(t-s)^{2 \alpha-2} E\left|x_{n}(s)-x_{n}\left(s-\frac{1}{n}\right)\right|^{2} d s
$$

Combining with the estimate for $I_{1}$ and $I_{2}$, it is derived that

$$
\begin{aligned}
E\left|x(t)-x_{n}(t)\right|^{2} \leq & \frac{k(T+1)}{\Gamma(\alpha)^{2}} \int_{0}^{t}(t-s)^{2 \alpha-2} E\left|x(s)-x_{n}(s)\right|^{2} d s \\
& +\frac{k T^{2 \alpha-1}}{(2 \alpha-1) \Gamma(\alpha)^{2}} \int_{0}^{t} E\left|x_{n}(s)-x_{n}\left(s-\frac{1}{n}\right)\right|^{2} d s \\
& +\frac{k}{\Gamma(\alpha)^{2}} \int_{0}^{t}(t-s)^{2 \alpha-2} E\left|x_{n}(s)-x_{n}\left(s-\frac{1}{n}\right)\right|^{2} d s,
\end{aligned}
$$


by Lemma 4.2 , if $s \geq \frac{1}{n}$, then

$$
E\left|x_{n}(s)-x_{n}\left(s-\frac{1}{n}\right)\right|^{2} \leq \frac{C}{n^{2 \alpha-1}},
$$

otherwise if $0 \leq s<\frac{1}{n}$,

$$
E\left|x_{n}(s)-x_{n}\left(s-\frac{1}{n}\right)\right|^{2}=E\left|x_{n}(s)-x_{n}(0)\right|^{2} \leq C s^{2 \alpha-1} \leq \frac{C}{n^{2 \alpha-1}}
$$

Following Eq. (4.3), we have

$$
\begin{aligned}
E\left|x(t)-x_{n}(t)\right|^{2} \leq & \frac{k(T+1)}{\Gamma(\alpha)^{2}} \int_{0}^{t}(t-s)^{2 \alpha-2} E\left|x(s)-x_{n}(s)\right|^{2} d s \\
& +\frac{k T^{2 \alpha-1}}{(2 \alpha-1) \Gamma(\alpha)^{2}} \int_{0}^{t} E\left|x_{n}(s)-x_{n}\left(s-\frac{1}{n}\right)\right|^{2} d s \\
& +\frac{T}{\Gamma(\alpha)^{2}} \int_{0}^{t}(t-s)^{2 \alpha-2} E\left|x_{n}(s)-x_{n}\left(s-\frac{1}{n}\right)\right|^{2} d s \\
\leq & \frac{k(T+1)}{\Gamma(\alpha)^{2}} \int_{0}^{t}(t-s)^{2 \alpha-2} E\left|x(s)-x_{n}(s)\right|^{2} d s \\
& +\frac{k T^{2 \alpha-1}(1+T)}{(2 \alpha-1) \Gamma(\alpha)^{2}} \frac{1}{n^{2 \alpha-1}} \\
= & : q_{1} \int_{0}^{t}(t-s)^{2 \alpha-2} E\left|x(s)-x_{n}(s)\right|^{2} d s+q_{2} .
\end{aligned}
$$

Applying Lemma 2.1, we obtain

$$
E\left|x(t)-x_{n}(t)\right|^{2} \leq q_{2}\left(1+E_{2 \alpha-1,1}\left(q_{1} \Gamma(2 \alpha-1) T^{2 \alpha-1}\right)\right)=: \frac{C}{n^{2 \alpha-1}} .
$$

This completes the proof.

Remark 4.1 When $\alpha=1$, i.e. Eq. (1.1) becomes a stochastic differential equation, the convergent rate of the scheme in Theorem 4.1 coincides with the well-known convergent rate of the classical Caratheodory results; see [23].

\section{Acknowledgements}

The first author is partially supported by National Science Foundation of China (11926322; 11801575) and "the Fundamental Research Funds for the Central Universities", South-Central University for Nationalities (Grant Number: CZY20014). The second author is partially supported by National Science Foundation of China (61876192) and "the Fundamental Research Funds for the Central Universities", South-Central University for Nationalities (Grant Number: KTZ20051; CTZ20020). The third author is partially supported by National Science Foundation of China (11901584) and "the Fundamental Research Funds for the Central Universities", South-Central University for Nationalities (Grant Number: CZY20013).

Funding

National Science Foundation of China (11926322; 11801575; 61876192; 11901584), "the Fundamental Research Funds for the Central Universities", South-Central University for Nationalities (Grant Number: KTZ20051; CTZ20020; CZY20014; CZY20013). 


\section{Competing interests}

The authors declare that they have no competing interests.

\section{Authors' contributions}

The authors contributed equally to this paper. All authors read and approved the final manuscript.

\section{Publisher's Note}

Springer Nature remains neutral with regard to jurisdictional claims in published maps and institutional affiliations.

Received: 6 July 2020 Accepted: 30 September 2020 Published online: 12 November 2020

\section{References}

1. Bandyopadhyay, B., Kamal, S.: Stabilization and Control of Fractional Order Systems: A Sliding Mode Approach. Springer, Switzerland (2015)

2. Diethelm, K.: The Analysis of Fractional Differential Equations: An Application-Oriented Exposition Using Differential Operators of Caputo Type. Springer, Berlin (2010)

3. Podlubny, I.: Fractional Differential Equations: An Introduction to Fractional Derivatives, Fractional Differential Equations, to Methods of Their Solution and Some of Their Applications. Elsevier, Netherland (1998)

4. Kilbas, A., Srivastava, H., Trujillo, J.: Theory and Applications of Fractional Differential Equations. Elsevier, Netherland (2006)

5. Povstenko, Y.: Fractional heat conduction equation and associated thermal stress. J. Therm. Stresses $28,83-102$ (2004)

6. Chen, W., Pang, G.: A new definition of fractional Laplacian with application to modeling three-dimensional nonlocal heat conduction. J. Comput. Phys. 309, 350-367 (2016)

7. Chen, W., Liang, Y., Hu, S., Sun, H.: Fractional derivative anomalous diffusion equation modeling prime number distribution. Power 21, 13 (2015)

8. Zhou, Y., Jiao, F.: Existence of mild solutions for fractional neutral evolution equations. Comput. Math. Appl. 59, 1063-1077 (2010)

9. Zou, G., Wang, B., Zhou, Y.: Existence and regularity of mild solutions to fractional stochastic evolution equations. Math. Model. Nat. Phenom. 13, 15 (2018)

10. Wang, J., Zhou, Y.: Existence and controllability results for fractional semilinear differential inclusions. Nonlinear Anal., Real World Appl. 12, 3642-3653 (2011)

11. Sakthivel, R., Revathi, P., Ren, Y.: Existence of solutions for nonlinear fractional stochastic differential equations. Nonlinear Anal., Theory Methods Appl. 81, 70-86 (2013)

12. Zhou, Y., Jiao, F.: Existence of mild solutions for fractional neutral evolution equations. Comput. Math. Appl. 59, $1063-1077(2010)$

13. Conga, N., Doanab, T., SiegmundcH, S., Tuan, T.: On stable manifolds for planar fractional differential equations. Appl. Math. Comput. 226, 157-168 (2014)

14. $\mathrm{Xu}, \mathrm{W} ., \mathrm{Xu}, \mathrm{W}$., Zhang, S.: The averaging principle for stochastic differential equations with Caputo fractional derivative. Appl. Math. Lett. 93, 79-84 (2019)

15. Wang, Y., Xu, J., Kloeden, P.: Asymptotic behavior of stochastic lattice systems with a Caputo fractional time derivative. Nonlinear Anal., Theory Methods Appl. 135, 205-222 (2016)

16. Doan, T., Kloeden, P., Huong, P., Tuan, H.: Asymptotic separation between solutions of Caputo fractional stochastic differential equations. Stoch. Anal. Appl. 36, 654-664 (2018)

17. Doan, T., Huong, P., Kloeden, P.: Euler-Maruyama scheme for Caputo stochastic fractional differential equations. J. Comput. Appl. Math. 112989, 1-26 (2020)

18. Zhou, Y., Wang, J., Zhang, L.: Basic Theory of Fractional Differential Equations. World scientific, Singapore (2016)

19. Zhou, Y., Peng, L.: Weak solutions of the timefractional Navier-Stokes equations and optimal control. Comput. Math. Appl. 73, 1016-1027 (2017)

20. Zhou, Y., Peng, L.: On the time-fractional Navier-Stokes equations. Comput. Math. Appl. 73, 874-891 (2017)

21. Zou, G.: A Galerkin finite element method for time-fractional stochastic heat equation. Comput. Math. Appl. 75 , 4135-4150 (2018)

22. Zou, G., Lv, G., Wu, J.: Stochastic Navier-Stokes equations with Caputo derivative driven by fractional noises. J. Math. Anal. Appl. 461, 595-609 (2018)

23. Mao, X.: Stochastic Differential Equations and Their Applications. Horwood, Chichester (2008)

24. Ye, H., Gao, J., Ding, Y.: A generalized Gronwall inequality and its application to a fractional differential equation. J. Math. Anal. Appl. 328, 1075-1081 (2007)

25. Wang, W., Chen, S., Guo, Z., Yan, X.: A note on the continuity for Caputo fractional stochastic differential equations. Chaos 30, $073106(2020)$ 